\title{
Timing of surgery following chemoradiotherapy in rectal Cancer
}

In this edition, the "Down-staging following neoadjuvant chemo-radiotherapy (NACTRT) for locally advanced rectal cancer - does the timing of surgery really matter?" aims to look at a topical issue. A retrospective analysis was undertaken of 110 patients. The authors reported a wide variation in the timing of surgery following completion of NACTRT, 4-474 days (median: 64.5). Patients were divided into those having surgery within 60 days $(n=42)$ or thereafter $(n=68)$. The authors demonstrated no difference in pathological variables (pathological complete response, tumor regression grade, circumferential resection margin status, lymph nodes or lymphovascular invasion) between the two groups. They also concluded that there was no significant difference in sphincter-saving surgery $(62 \%$ vs. 53\%) or early recurrence rates (median follow-up 13 months (range: 4-27).

A study such as this, with a small sample size, lack of power and retrospective analysis limit the conclusions but raise some interesting issues. To understand whether or not the timing of surgery after radiotherapy alters practice-there needs to be a comparison between the pretreatment tumor $(\mathrm{T})$ and nodal $(\mathrm{N})$ stage with the pathological specimen, or posttreatment imaging - hence, we are not really sure where and how "down-staging" occurs. Crucially, if no restaging after chemo/radiotherapy takes place then it is highly unlikely that the surgical treatment plan will be altered. Thus, it is not possible to infer what effects may, or may not have on sphincter saving surgery or early local recurrence. However, the observation of an extremely wide variation in timing of surgery in the longer interval group, from 61 to 474 , adds to the body of previous published evidence that there is a wide variation in the scheduling of surgery following preoperative treatment. ${ }^{[1]}$ This variation is in part attributable to patient choice and/or waiting list problems. However, the lack of clarity regards the standard of care in timing of surgery following completion of preoperative chemo/radiotherapy is once again highlighted.

\begin{tabular}{|l|l|}
\hline \multicolumn{3}{|c|}{ Access this article online } \\
\hline Quick Response Code: & Website: \\
\hline & www.jmpo.org \\
\hline & \\
\hline
\end{tabular}

Preoperative chemo/radiotherapy for rectal cancer is used to reduce tumor stage and size, thus facilitating complete resection and reducing the incidence of local recurrence. Potentially, greater tumor downsizing may allow greater rates of sphincter saving surgery and reduce the need for permanent stomas. In 1999, Francois et al. randomized 201 patients to surgery at 2 or 6 weeks following completion of radiotherapy. The longer interval group had better tumor response and pathological down-staging and higher sphincter saving surgery rates. ${ }^{[2]}$ Since the Lyon R90-01 trial, surgeons have operated at approximately 6-8 weeks after the completion of preoperative therapy. More recent studies have observed ongoing down-staging and better surgical outcomes with a delay of longer than 8 weeks. ${ }^{[3,4]}$ Ideally, surgery should take place at the time of maximal response to the radiotherapy, the effects of which are known to be time dependent.

In our own cancer networks published retrospective review of rectal cancers treated preoperatively over a 27 -month period, $32(34 \%)$ patients underwent surgery at 6-8 weeks, $45(47 \%)$ at $>8$ weeks and $18(19 \%)$ at $<6$ weeks after radiotherapy. ${ }^{[1]}$ Delay was attributed to scheduling in $87 \%$ of cases and comorbidities in the remainder. Tumor downstaging occurred in $6(33.3 \%)$ patients in $<6$ weeks group, $12(37.5 \%)$ in 6-8 weeks group, and $28(62.2 \%)$ for $>8$ weeks with no significant differences in perioperative morbidity. More patients were staged ypT0-T2, 19/45 $(42 \%)$ in the $>8$ weeks group versus other groups, $14 / 50$ $(28 \%, P<0.05)$. We therefore concluded that following chemo/radiotherapy, surgery frequently occurs $>8$ weeks and is associated with increased downstaging, but that the consequences on survival and perioperative morbidity warranted further investigation.

Post-NACTRT both circumferential resection margin and tumor stage have a direct correlation with the likelihood of local recurrence. ${ }^{[5]}$ With a number of studies demonstrating an ongoing response to preoperative therapy beyond the 6-8 weeks window, ${ }^{[3,4]}$ it seems logical that surgery prior to 8 weeks maybe premature, in all but those who have progressive disease on interim scans. Those patients with a complete pathological response following chemo/ radiotherapy for rectal cancer have a significantly better long term outcome. ${ }^{[5,6]}$ The presented study was not powered to examine the relationship between pathological complete remission $(\mathrm{CR})(n=22)$ and timing of surgery. 
However, more patients in the delayed group ( $>60$ days) had a pathological CR (23\% vs. 14\%). Previous work has shown that a greater delayed to surgery following completion of preoperative therapy is associated with an increased likelihood of achieving a pathological CR. ${ }^{[6]}$

There is sufficient published evidence to suggest that there is ongoing local down-staging beyond 8 weeks in many patients. However, while some centers have now moved to routinely operating with longer delays, there is a concern that such delays cause deferral of systemic chemotherapy, and create the risk of inadvertent progression of systemic disease. Hence, a number of key questions need to be addressed before any change in the standard of care can occur. First, does a delay to surgery improve local recurrence rates? Is there an increase in surgical complications, and if so does this result in a delay to adjuvant therapy? With a longer interval, is there an increased risk of disease progression, either local or metastatic? As highlighted by this study, only with a prospective randomized controlled multicenter study with strict adherence to the timing of surgery can level I evidence be provided that can finally answer these questions and controversy removed from an area of such clinical and academic importance.

With this in mind the Royal Marsden Hospital designed and launched the 6 versus 12 study (http://www.clinical trials. gov/show/NCT01037049) and the French trial (Greccar 6) was designed, randomizing between 7 and 11 weeks (http:/ / www.clinicaltrials.gov/show/NCT01648894). These multicenter prospective randomized controlled trials are powered with the primary aim of determining whether greater rectal cancer down-staging and regression occurs with a greater delay to surgery after completion of chemo/ radiotherapy. The secondary aims include sphincter saving surgery rates, early surgical complications, diversion stoma rates positron emission tomography-computed tomography and magnetic resonance imaging monitoring and response, radiotherapy-related bowel toxicity and recurrence free survival.

The 6 versus 12 study and Greccar trials will be the first prospective randomized studies examining the optimum time interval between completion of chemo/radiotherapy and surgery in the treatment of locally advanced rectal cancer since the Lyon R90-01 trial in 1999. The potential impact of identifying the optimum time to operate will be to the individual patient, the health care provider and future clinical trials. By identifying the time of maximal response local recurrence and sphincter saving surgery rates and hence functional outcome may be improved. This will add to the potentially reduced cost implications to healthcare providers along with clarity for scheduling of surgery. The establishment of a "gold standard" in the timing of surgery following NACTRT for locally advanced rectal cancer, will add the design of future trials by removing a variable that currently exists.

Gina Brown, Jessica Evans

Department of Radiology, Royal Marsden NHS Foundation Trust Sutton, Surrey, SM2 5PT, Sutton, United Kingdom E-mail: gina.brown@rmh.nhs.uk

\section{REFERENCES}

1. Evans J, Tait D, Swift I, Pennert K, Tekkis P, Wotherspoon A, et al. Timing of surgery following preoperative therapy in rectal cancer: The need for a prospective randomized trial? Dis Colon Rectum 2011;54:1251-9.

2. Francois $Y$, Nemoz CJ, Baulieux J, Vignal J, Grandjean JP, Partensky C, et al. Influence of the interval between preoperative radiation therapy and surgery on downstaging and on the rate of sphincter-sparing surgery for rectal cancer: The Lyon R90-01 randomized trial. J Clin Oncol 1999;17:2396.

3. Johnston DF, Lawrence KM, Sizer BF, Arulampalam TH, Motson RW, Dove E, et al. Locally advanced rectal cancer: Histopathological correlation and predictive accuracy of serial MRI after neoadjuvant chemotherapy. $\mathrm{Br} \mathrm{J}$ Radiol 2009; 82:332-6.

4. Kerr SF, Norton S, Glynne-Jones R. Delaying surgery after neoadjuvant chemoradiotherapy for rectal cancer may reduce postoperative morbidity without compromising prognosis. Br J Surg 2008;95:1534-40.

5. Maas M, Nelemans PJ, Valentini V, Das P, Rödel C, Kuo LJ, et al. Long-term outcome in patients with a pathological complete response after chemoradiation for rectal cancer: A pooled analysis of individual patient data. Lancet Oncol 2010;11:835-44.

6. de Campos-Lobato LF, Geisler DP, da Luz Moreira A, Stocchi L, Dietz D, Kalady MF. Neoadjuvant therapy for rectal cancer: The impact of longer interval between chemoradiation and surgery. J Gastrointest Surg 2011;15:444-50.

How to cite this article: Brown G, Evans J. Timing of surgery following chemoradiotherapy in rectal Cancer. Indian J Med Paediatr Oncol 2014;35:235-6.

Source of Support: Nil, Conflict of Interest: None declared. 